\title{
Factors Associated with Low Socioeconomic Status Predict Poor Postoperative Follow-up after Meningioma Resection
}

\author{
Arash Nayeri ${ }^{1}$ Philip R. Brinson ${ }^{1}$ Kyle D. Weaver ${ }^{1}$ Reid C. Thompson ${ }^{1}$ Lola B. Chambless ${ }^{1}$ \\ ${ }^{1}$ Department of Neurosurgery, Vanderbilt University Medical Center, \\ Nashville, Tennessee, United States \\ Address for correspondence Arash Nayeri, Department of \\ Neurosurgery, Vanderbilt University School of Medicine, 201 Light \\ Hall, Nashville, TN 37232, United States \\ J Neurol Surg B 2016;77:226-230. \\ (e-mail: Arash.nayeri@vanderbilt.edu).
}

\begin{abstract}
Keywords

- meningioma

- follow-up

- socioeconomic status

- tobacco

Objectives To quantify the rates of loss of follow-up after meningioma resection and to identify any key demographical associations.

Design Retrospective cohort.

Setting Vanderbilt University Medical Center, 2001-2013.

Participants A total of 281 patients surgically treated for an intracranial meningioma at a single institution between 2001 and 2013.

Main Outcome Measures Patient clinical follow-up within the first postoperative year. Results A history of tobacco use $(p<0.0001)$, ongoing alcohol abuse at time of presentation $(p=0.0014)$, Medicaid coverage $(p<0.0001)$, and lack of a college degree $(p<0.0001)$ were all found to be predictors of loss of follow-up at a statistically significant level.

Conclusions Several factors associated with low socioeconomic status are predictors of poor clinical follow-up after meningioma resection. The health risk of poor follow-up in this patient population is significant, and increased measures are needed to ensure regular appointment attendance.
\end{abstract}

\section{Introduction}

Poor patient compliance is a challenge faced in all subspecialties of medicine. Particular attention has been paid to the issue of nonattendance at clinic appointments. ${ }^{1-4}$ Previous authors have attempted to quantify the rates of appointment nonattendance in various medical subspecialties. Low socioeconomic status (SES), Medicaid coverage, ethnic minority group status, unemployment, and poor social support systems have repeatedly been shown to be associated with higher rates of missed appointments. ${ }^{5-8}$ Several articles, particularly those focused on the care of patients with psychiatric disease, have identified substance abuse in addition to mood and anxiety disorders as other strong predictors of poor follow-up. ${ }^{9-13}$

received

May 16, 2015

accepted

September 8, 2015

published online

October 28, 2015

Regardless of the cause of nonattendance and the demographical associations with such behavior, the costs of missed clinical appointments are well documented. The financial burden of appointment nonattendance and poor follow-up has been cited as a significant source of waste and a potential target for financial savings in the health care system. ${ }^{14-16}$ Moreover, missed appointments have been shown to strain the patient-provider relationship and are a common complaint by health care providers. ${ }^{17}$

We sought to quantify the rate of missed follow-up appointments after the resection of an intracranial meningioma at our institution. We examined the first postoperative year to study the patterns and rates of clinical follow-up. We aim to show key demographical associations with poor rates of follow-up in this patient population.

(c) 2016 Georg Thieme Verlag KG Stuttgart · New York
DOI http://dx.doi.org/ 10.1055/s-0035-1566122. ISSN 2193-6331. 


\section{Methods}

\section{Patient Population}

We conducted a retrospective cohort study on 281 patients surgically treated for an intracranial meningioma at a single institution between 2001 and 2013. For patients with a history of multiple meningioma resections at our institution, only data with regard to the initial resection were used in the study. Four attending neurosurgeons with specialized training in neurosurgical oncology performed all resections.

\section{Data Collection and Study Variables}

Records of clinical and radiographic data were retrospectively obtained from the electronic medical record and entered into a REDCap (Research Electronic Data Capture) database. ${ }^{18}$ All such entries in the medical record were reviewed from the time of operation to the date of the last chart review, March 30, 2015. Patients who indicated postoperative follow-up away from our institution were excluded from the study. Patient follow-up, age, gender, race, distance from our institution, educational attainment, insurance status, history of illicit substance and tobacco use, continued alcohol abuse, extent of surgical resection, and pathologic grade were recorded for each patient.

A loss of follow-up variable was created based on patient clinical follow-up within the first postoperative year. Clinical follow-up was assessed at neurosurgery, radiation oncology, or neuro-oncology clinic visits. Failure to show up at designated follow-up appointments within the first postoperative year was assigned as the general definition of the variable. Patients who missed a postoperative clinic appointment but returned for a makeup appointment within 6 months of the original appointment date were not designated as lost to follow-up. Patients who sent reports of routine postoperative imaging away from our institution were not designated as lost to follow-up.

Patient age was defined as age at time of operation. The race and gender variables were recorded from the social history at the time of the initial clinical evaluation. Distance from our institution was calculated based on patient address at the time of surgery. Educational attainment was defined as the highest level of reported education at the time of operation; subcategories were created based on patient graduation from high school or the attainment of high school equivalency, any history of college enrollment, graduation from college, and attainment of a graduate degree. Insurance status was assayed at time of operation as private insurance, Medicare, Medicaid, VA/Tricare, or uninsured. History of illicit substance abuse was defined as any admission to a history of intravenous (IV) or illicit drug use on behalf of the patient at the time of preoperative assessment. Similarly, history of tobacco use was defined as any admission to a history of tobacco use at the time of preoperative assessment. Continued alcohol abuse was defined as current consumption of more than two alcoholic drinks per day, self-report of current alcohol abuse, or a positive answer to a question on the CAGE inquiry (screening method for alcoholism) at the time of the preoperative assessment. Extent of resection was classified as gross total resection or incomplete resection based on the interpretation of the immediate postoperative magnetic resonance imaging scan by the neuroradiologist. Tumor grade was assigned as World Health Organization (WHO) grade I, II, or III based on the neuropathologist's assessment of tumor subsequent to the resection.

\section{Statistical Analysis}

All information was de-identified prior to statistical analysis in Microsoft Excel and JMP Pro. ${ }^{19,20}$ To determine any associations between loss of follow-up and continuous variables used as predictors in the study-age and distance from our institution-the Pearson correlation was used. The Fisher exact test was used to test for any association between race, gender, educational attainment, insurance status, history of illicit substance and tobacco use, or continued alcohol abuse with loss of follow-up. All results were deemed to be statistically significant if $p<0.05$.

\section{Results}

A total of 281 patients were evaluated in this study. The mean age at time of operation was $51.4 \pm 14.5$ years. Overall, 195 (69\%) of the patients were female; 244 (87\%) were white. Mean distance from patient residence to our institution at the time of operation was $92.1 \pm 70.1$ miles. Together, private insurance (125 patients [44\%]) and Medicare (110 patients [39\%]) represented the insurance coverage of most of the patients. A total of 82 patients (29\%) were college graduates; 149 (53\%) did not have a college degree. We could not determine the education attainment of 50 patients (18\%). - Table 1 provides a demographic overview.

Overall, 95 patients (34\%) admitted to a history of tobacco use, either ongoing at the time of preoperative evaluation or in the past. A total of 39 patients (14\%) identified ongoing alcohol abuse at the time of the preoperative evaluation. Twenty-eight patients (10\%) were covered by Medicaid at the time of operation. A history of tobacco use, ongoing alcohol abuse at time of presentation, Medicaid coverage, and lack of a

Table 1 Demographics of patients

\begin{tabular}{|l|l|}
\hline $\begin{array}{l}\text { Mean age at time of } \\
\text { operation, years }\end{array}$ & 51.4 (SD: 14.5) \\
\hline Gender & $\begin{array}{l}195 \text { female (69\%); 86 male } \\
(31 \%)\end{array}$ \\
\hline Race & $\begin{array}{l}244 \text { white (87\%); 28 African } \\
\text { American } \\
(10 \%) ; 6 \text { Hispanic } \\
(2 \%) ; 3 \text { Asian (1\%) }\end{array}$ \\
\hline $\begin{array}{l}\text { Average distance between } \\
\text { patient residence and } \\
\text { imaging center, miles }\end{array}$ & 92.1 (SD: 70.1) \\
\hline Insurance & $\begin{array}{l}125 \text { private (44\%); 110 } \\
\text { Medicare (39\%); 28 Medicaid } \\
(10 \%) ; 11 \text { VA/Tricare (4\%); } \\
7 \text { uninsured (2\%) }\end{array}$ \\
\hline
\end{tabular}

Abbreviation: SD, standard deviation. 
college degree were all found to be predictors of loss of follow-up at a statistically significant level. These findings are summarized in - Table 2 and -Fig. 1.

Five patients (2\%) claimed any history of illicit or IV drug abuse. Neither a history of IV nor illicit drug use, age, gender, race, distance of patient residence from our institution, or insurance coverage (except for Medicaid) was found to be significantly associated with a different rate of followup. - Table 3 summarizes these results.

Overall, 53 patients (19\%) demonstrated loss of follow-up within the first postoperative year. Of these 53 patients, 16 (30\%) had tumor pathology concerning for atypical meningioma (either WHO grade II or III). Eleven patients (21\%) with loss of follow-up had incomplete resections of their meningioma. A total of 22 patients (43\%) with loss of follow-up had either an atypical tumor pathology or an incomplete resection.

\section{Discussion}

The challenge of appointment nonattendance continues to vex physicians across all specialties and is a continued source of waste in our health care system. Previous authors have noted the rates of poor follow-up in different patient groups, identified SES and other demographical associations with this behavior, and provided cost estimates for appointment nonattendance.

In this analysis of 281 patients with a resected meningioma at our institution, we identified a very high rate of
Table 3 Factors not associated with loss of follow-up at a statistically significant level ${ }^{\mathrm{a}}$

\begin{tabular}{|l|l|}
\hline Variable & $p$ value \\
\hline Increased age & 0.501 \\
\hline Male gender & 0.136 \\
\hline Race (African American) & 0.134 \\
\hline Race (Hispanic) & 1.00 \\
\hline Uninsured & 0.622 \\
\hline History of illicit substance use & 0.239 \\
\hline Distance from imaging center & 0.956 \\
\hline
\end{tabular}

aPatient age, gender, race, insurance status (except for Medicaid), history of illicit substance abuse, and distance of residence from imaging center were not associated with different rates of postoperative follow-up.

appointment nonattendance within the first postoperative year. We quantified the rate of loss of follow-up within a year of the operation at $19 \%$, within range of estimates for other patient populations in previous studies. ${ }^{1,2,4}$ Of note, we found significantly higher rates of poor follow-up patterns that we did not quantify in our study. In particular, a significant number of patients were either late for their appointments, missed appointments without proper notification, or had to reschedule for a new clinic visit after having missed an

Table 2 Factors associated with increased rates of loss of follow-up ${ }^{a}$

\begin{tabular}{|l|l|l|l|}
\hline Variable & Patient prevalence (\%) & Relative risk & $p$ value \\
\hline History of tobacco use & $95(34)$ & 4.9 & $<0.0001$ \\
\hline Current alcohol abuse & $39(14)$ & 2.5 & 0.0014 \\
\hline Medicaid coverage & $28(10)$ & 3.7 & $<0.0001$ \\
\hline Lack of a college degree & $149(53)$ & 3.9 & $<0.0001$ \\
\hline
\end{tabular}

${ }^{a}$ History of tobacco use, current alcohol abuse, Medicaid insurance, and lack of a college degree were all associated with increased rates of poor follow-up.
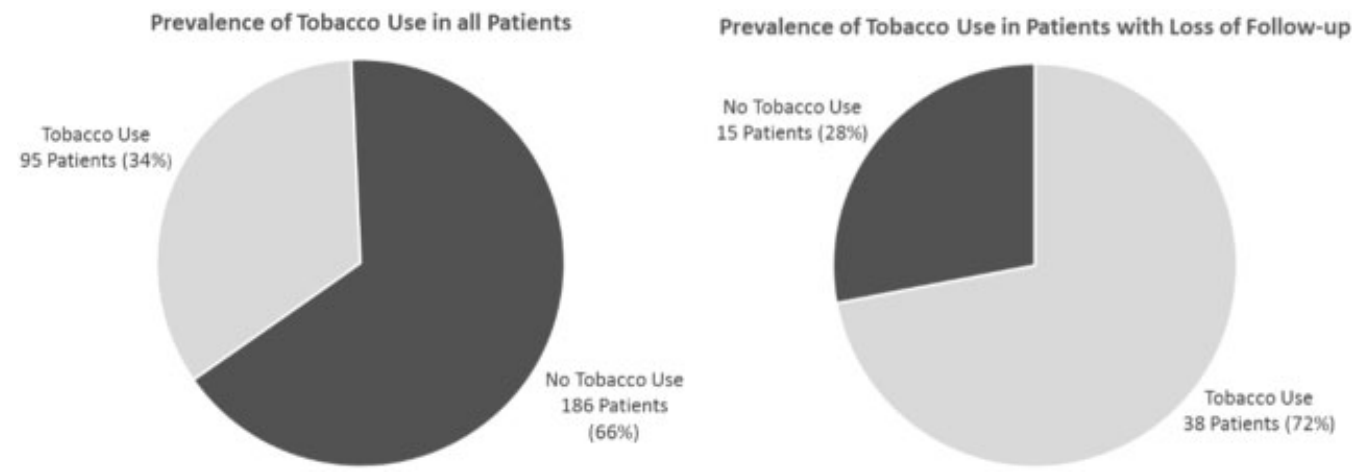

Fig. 1 Increased rate of tobacco use among patients with loss of follow-up. A history of tobacco use was reported in $34 \%$ of all patients included in the study. Among the subgroup with loss of follow-up, a history of tobacco use was noted in $72 \%$ of patients. Tobacco use was most predictive of loss of follow-up (relative risk: 4.9). 
appointment. Furthermore, although we did not quantify the rates of follow-up after the first postoperative year, we noticed a significant number of patients who were lost to follow-up $>1$ year after their operation. Overall, the rates of poor follow-up and eventual loss of follow-up in our patient population is in fact much higher than the $19 \%$ we reported for our given criteria in this study.

We studied several previously identified demographic factors associated with poor follow-up in other patient populations. Our data indicate that any smoking history, current alcohol abuse, lack of a 4-year college education, and Medicaid as the primary insurance are associated with a significantly higher risk of postoperative loss of follow-up. Such factors have previously been identified in several other patient groups to be associated with poor follow-up and are all in line with lower SES. ${ }^{21-23}$

Lower SES has been repeatedly correlated with poor health outcomes and difficulty with access to health care. Gallo and Matthews developed the Reserve Capacity Model in 2003 as a framework to examine the psychological factors in SESdriven health disparities. ${ }^{24,25}$ Their groundbreaking study noted indirect evidence of relationships between low SES and negative emotions and cognitions, low SES and targeted health outcomes, and negative emotions and cognitions with poor health outcomes. Low SES and the accompanying negative cognitions and emotions have been associated with poor health outcomes and difficulty with health care access in several different health care settings. ${ }^{26-28}$ Our study noted several factors related to low SES that were associated with poor follow-up in our patient population. We are able to extend this proposed relationship between low SES and irregular access to health care to the patient population with a history of resected meningioma. Furthermore, it is essential to take into the account the innumerable economic and social challenges that preferentially affect patients of lower SES when considering the cause of appointment nonattendance. A certain degree of understanding and empathy may be due in particular patient situations with appointment nonattendance. Of note, focusing the blame of missed appointments on patients and a negative view of such patients on behalf of the clinical staff has been shown to be detrimental to both the staff and the patients. ${ }^{17}$

Although not quantified in our study, patients clearly take health risks by failing to follow up after their tumor resection. Of the 53 patients with loss of follow-up, 16 (30\%) had pathology indicative of an atypical meningioma (WHO grade II or III). Atypical meningiomas are well known for an increased risk of recurrence, up to $40 \%$ at 5 years. ${ }^{29-33}$ Moreover, 11 patients (21\%) with loss of follow-up had an incomplete resection of their meningioma and are also at increased risk for tumor recurrence. ${ }^{34}$ Of the 53 patients who were noted to have been lost to follow-up within the first postoperative year, 22 (43\%) had either an incomplete resection or atypical tumor pathology. For these patients in particular, the lack of appropriate clinical and radiologic follow-up is very concerning. Moreover, the lack of appropriate postoperative follow-up can lead to eventual presentation with larger tumor sizes. The association between increased tumor size and poor prognosis is also well established. ${ }^{34-36}$ Overall, the health risk to which patients predispose themselves to with poor clinical follow-up after meningioma resection is of great concern.

Admittedly, finding an effective solution to the challenge of appointment nonattendance is an ongoing effort. Several different interventions on behalf of clinicians have been reviewed in the literature with varying degrees of efficacy in remedying this problem. More recently, however, a large number of studies have identified mobile-phone short message service (SMS) reminders to be an effective and low-cost means of reducing the rate of appointment nonattendance. The use of SMS text reminders has been tried in a variety of different clinical settings with a promising reduction in rates of nonattendance. ${ }^{37-40}$ We think that SMS reminders should be considered as a potential tool in improving the rates of follow-up in patients with a history of resected meningioma.

One trend we noticed during our review of clinic follow-up rates was scheduling subsequent appointments many months in advance. We realize the clinic schedules for the neurosurgeons, radiation oncologists, and neuro-oncologists who manage patients with meningiomas are very busy, and booking appointments in advance has become commonplace. However, appointments made many months in advance have been repeatedly associated with higher rates of nonattendance across various medical specialties. ${ }^{41}$ Instead, patientfocused booking and booking appointments closer to the scheduled date of return are advocated as an effective way to reduce the rate of appointment nonattendance. It is very likely, however, that a complete transition to patient-focused booking and booking appointments more closely to the schedule date may not be feasible. Nevertheless, we believe that such changes in appointment scheduling are particularly warranted in patients with a history of incomplete resections or atypical meningiomas because these patients carry a significantly increased risk for tumor recurrence and may benefit the most from regular follow-up.

\section{Conclusions}

Our study demonstrates a marked rate of loss of clinical follow-up within the first postoperative year after the resection of a meningioma. In our analysis, patients with any smoking history, active alcohol abuse, lack of a college degree, and those covered by Medicaid were more likely to demonstrate such a pattern of poor follow-up. Poor clinical follow-up in patients of lower SESs was previously noted in various different patient populations. In patients with incomplete resections or atypical tumor pathologies, poor postoperative follow-up is particularly concerning due to these individuals' high risk of tumor recurrence. SMS reminders and patientfocused appointment booking are two well-studied interventions that we advocate for patients with a history of meningioma resection. Further studies are needed to characterize the barriers to regular follow-up in these patients and to identify better ways to remedy the challenge of appointment nonattendance. 


\section{Acknowledgments}

The authors wish to acknowledge Marc Prablek and Diana Douleh for their assistance in the collection of the clinical database used for this study.

\section{References}

1 George A, Rubin G. Non-attendance in general practice: a systematic review and its implications for access to primary health care. Fam Pract 2003;20(2):178-184

2 Waller J, Hodgkin P. Defaulters in general practice: who are they and what can be done about them? Fam Pract 2000;17(3):252-253

3 Compton MT, Rudisch BE, Craw J, Thompson T, Owens DA. Predictors of missed first appointments at community mental health centers after psychiatric hospitalization. Psychiatr Serv 2006;57(4):531-537

4 Chariatte V, Berchtold A, Akré C, Michaud PA, Suris JC. Missed appointments in an outpatient clinic for adolescents, an approach to predict the risk of missing. J Adolesc Health 2008;43(1):38-45

5 Brown KA, Shetty V, Delrahim S, Belin T, Leathers R. Correlates of missed appointments in orofacial injury patients. Oral Surg Oral Med Oral Pathol Oral Radiol Endod 1999;87(4):405-410

6 Pesata V, Pallija G, Webb AA. A descriptive study of missed appointments: families' perceptions of barriers to care. J Pediatr Health Care 1999;13(4):178-182

7 Tanner EK, Feldman RH. Strategies for enhancing appointment keeping in low-income chronically ill clients. Nurs Res 1997;46(6): 342-344

8 Horsley BP, Lindauer SJ, Shroff B, et al. Appointment keeping behavior of Medicaid vs non-Medicaid orthodontic patients. Am J Orthod Dentofacial Orthop 2007;132(1):49-53

9 Howe CJ, Cole SR, Napravnik S, et al. The role of at-risk alcohol/drug use and treatment in appointment attendance and virologic suppression among HIV(+) African Americans. AIDS Res Hum Retroviruses 2014;30(3):233-240

10 Shan SJ, Zahurak M, Khan Z, Califano JA. Reluctance to undergo follow-up screening for head and neck cancer is associated with income, gender, and tobacco use. ORL J Otorhinolaryngol Relat Spec 2010;72(5):266-271

11 Kruse GR, Rohland BM, Wu X. Factors associated with missed first appointments at a psychiatric clinic. Psychiatr Serv 2002;53(9): 1173-1176

12 Ciechanowski P, Russo J, Katon W, et al. Where is the patient? The association of psychosocial factors and missed primary care appointments in patients with diabetes. Gen Hosp Psychiatry 2006;28(1):9-17

13 Cashman SB, Savageau JA, Lemay CA, Ferguson W. Patient health status and appointment keeping in an urban community health center. J Health Care Poor Underserved 2004;15(3):474-488

14 Stone CA, Palmer JH, Saxby PJ, Devaraj VS. Reducing non-attendance at outpatient clinics. J R Soc Med 1999;92(3):114-118

15 Breen R, Thornhill JT. Noncompliance with medication for psychiatric disorders. CNS Drugs 1998;9(6):457-471

16 Cruz M, Cruz RF. Compliance and costs in a case management model. Community Ment Health J 2001;37(1):69-77

17 Husain-Gambles M, Neal RD, Dempsey O, Lawlor DA, Hodgson J. Missed appointments in primary care: questionnaire and focus group study of health professionals. Br J Gen Pract 2004;54(499):108-113

18 Harris PA, Taylor R, Thielke R, Payne J, Gonzalez N, Conde JG. Research electronic data capture (REDCap)-a metadata-driven methodology and workflow process for providing translational research informatics support. J Biomed Inform 2009;42(2):377-381

19 Microsoft Excel. Redmond, WA: Microsoft Corporation; 2011

20 JMP Pro, v.11. Cary, NC : SAS Institute; 1989-2007

21 Winkleby MA, Jatulis DE, Frank E, Fortmann SP. Socioeconomic status and health: how education, income, and occupation con- tribute to risk factors for cardiovascular disease. Am J Public Health 1992;82(6):816-820

22 Foraker RE, Rose KM, Suchindran CM, Chang PP, McNeill AM, Rosamond WD. Socioeconomic status, Medicaid coverage, clinical comorbidity, and rehospitalization or death after an incident heart failure hospitalization: Atherosclerosis Risk in Communities cohort (1987 to 2004). Circ Heart Fail 2011;4(3):308-316

23 Livingston M. Socioeconomic differences in alcohol-related risktaking behaviours. Drug Alcohol Rev 2014;33(6):588-595

24 Gallo LC, Matthews KA. Understanding the association between socioeconomic status and physical health: do negative emotions play a role? Psychol Bull 2003;129(1):10-51

25 Gallo LC, Bogart LM, Vranceanu AM, Matthews KA. Socioeconomic status, resources, psychological experiences, and emotional responses: a test of the reserve capacity model. J Pers Soc Psychol 2005;88(2):386-399

26 Bennett KK, Buchanan DM, Jones PG, Spertus JA. Socioeconomic status, cognitive-emotional factors, and health status following myocardial infarction: testing the Reserve Capacity Model. J Behav Med 2015;38(1):110-121

27 Gallo LC, de Los Monteros KE, Shivpuri S. Socioeconomic status and health: what is the role of reserve capacity? Curr Dir Psychol Sci 2009;18(5):269-274

28 Matthews KA, Räikkönen K, Gallo L, Kuller LH. Association between socioeconomic status and metabolic syndrome in women: testing the reserve capacity model. Health Psychol 2008;27(5):576-583

29 Aghi MK, Carter BS, Cosgrove GR, et al. Long-term recurrence rates of atypical meningiomas after gross total resection with or without postoperative adjuvant radiation. Neurosurgery 2009;64(1): 56-60; discussion 60

30 Komotar RJ, Iorgulescu JB, Raper DM, et al. The role of radiotherapy following gross-total resection of atypical meningiomas. J Neurosurg 2012;117(4):679-686

31 Adeberg S, Hartmann C, Welzel T, et al. Long-term outcome after radiotherapy in patients with atypical and malignant meningiomas-clinical results in 85 patients treated in a single institution leading to optimized guidelines for early radiation therapy. Int J Radiat Oncol Biol Phys 2012;83(3):859-864

32 Aizer AA, Arvold ND, Catalano P, et al. Adjuvant radiation therapy, local recurrence, and the need for salvage therapy in atypical meningioma. Neuro-oncol 2014;16(11):1547-1553

33 Moon HS, Jung S, Jang WY, Jung TY, Moon KS, Kim IY. Intracranial meningiomas, WHO grade II: prognostic implications of clinicopathologic features. J Korean Neurosurg Soc 2012;52(1):14-20

34 Ayerbe J, Lobato RD, de la Cruz J, et al. Risk factors predicting recurrence in patients operated on for intracranial meningioma. $\mathrm{A}$ multivariate analysis. Acta Neurochir (Wien) 1999;141(9): 921-932

35 McCarthy BJ, Davis FG, Freels S, et al. Factors associated with survival in patients with meningioma. J Neurosurg 1998;88(5):831-839

36 Chan RC, Thompson GB. Morbidity, mortality, and quality of life following surgery for intracranial meningiomas. A retrospective study in 257 cases. J Neurosurg 1984;60(1):52-60

37 Guy R, Hocking J, Wand H, Stott S, Ali H, Kaldor J. How effective are short message service reminders at increasing clinic attendance? A meta-analysis and systematic review. Health Serv Res 2012; 47(2):614-632

38 Koshy E, Car J, Majeed A. Effectiveness of mobile-phone short message service (SMS) reminders for ophthalmology outpatient appointments: observational study. BMC Ophthalmol 2008;8:9

39 Sims H, Sanghara H, Hayes D, et al. Text message reminders of appointments: a pilot intervention at four community mental health clinics in London. Psychiatr Serv 2012;63(2):161-168

40 Car J, Ng C, Atun R, Card A. SMS text message healthcare appointment reminders in England. J Ambul Care Manage 2008;31(3):216-219

41 Tatham A, Murdoch I. The effect of appointment rescheduling on monitoring interval and patient attendance in the glaucoma outpatient clinic. Eye (Lond) 2012;26(5):729-733 